\title{
Radio Signatures of Sunspot NOAA 12192
}

\author{
Minttu Uunila, Juha Kallunki \\ Metsähovi Radio Observatory, Aalto University, Kylmälä, Finland \\ Email: minttu.uunila@aalto.fi
}

Received 27 September 2014; revised 25 October 2014; accepted 20 November 2014

Copyright (C) 2014 by authors and Scientific Research Publishing Inc.

This work is licensed under the Creative Commons Attribution International License (CC BY).

http://creativecommons.org/licenses/by/4.0/

(c) (i) Open Access

\begin{abstract}
In this paper, we present an overview of radio signatures of sunspot NOAA 12192 measured with various instruments with frequencies of $37 \mathrm{GHz}, 11.2 \mathrm{GHz}$ and $200-400 \mathrm{MHz}$ at Aalto University Metsähovi Radio Observatory (MRO). The data were observed during October 20 - 29, 2014. In total, 12 solar radio bursts at $11.2 \mathrm{GHz}$ and 8 at $200-400 \mathrm{MHz}$, with varying intensities and properties, were observed. Radio brightening was captured in several solar radio maps. NOAA 12192 is the largest observed sunspot during solar cycle 24. We show that this exceptional radio brightening belongs to the strongest category including less than $5 \%$ of radio brightenings ever measured at MRO.
\end{abstract}

\section{Keywords}

Solar Activity, Solar Flare, Solar Cycle, Solar Radio Burst

\section{Introduction}

Sunspot NOAA 12192, Figure 1, was both active and visible during October 20 - 29, 2014. It is the largest sunspot, with a diameter $>100,000 \mathrm{~km}$, measured in solar cycle 24 regarding the size of the umbra [1], [2], and also the largest one observed in 24 years [3] producing tens of solar events [4]. The high activity and unstability of NOAA 12192 shows that the sunspot has a highly complex magnetic structure. The sunspot, on the right side in Figure 1, shows also various flux structures measured with SDO/HMI magnetogram. The black areas have magnetic fields with negative polarity, and white areas have positive polarity. In typical sunspots, like 2194 in Figure 1 on the right side, there is only one negative and one positive polarity area, and they are clearly separated from each other. In the case of NOAA 12192, marked as 2192 in Figure 1, there are several polarity areas. The negative magnetic field in the Figure 1 is $-2.0 \mathrm{kG}$, and the positive magnetic field is more than $1.7 \mathrm{kG}$.

Large sunspots like NOAA 12192 are not usually observed, because they tend to lose their stability as they grow and change into more complex magnetic structure. They usually disintegrate into smaller sunspots accord- 

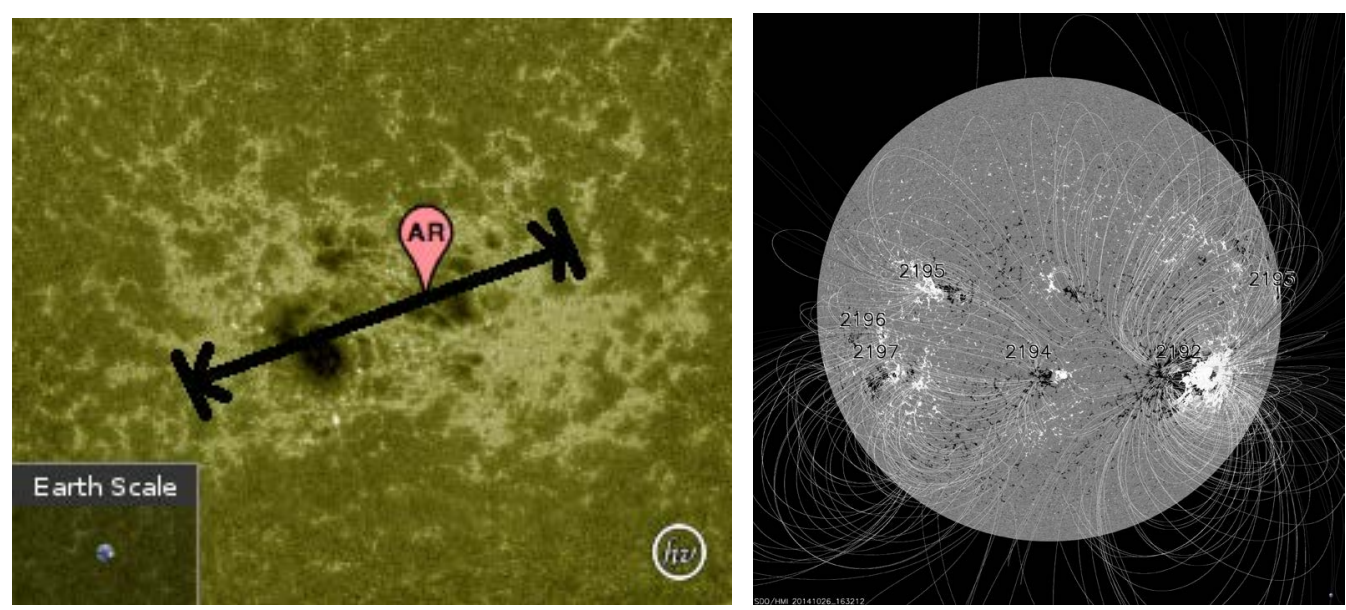

Figure 1. NOAA 12192 as seen by SDO AIA $1600 \AA$. Figure on the left is courtesy of NASA, ESA and JAXA. The marked area is $>100000 \mathrm{~km}$. Figure on the right with photosgraphic magnetic field map is measured by SDO/HMI magnetogram (at $6173 \AA$ ) on October 26, 2014 at 22:01:59 UT, courtesy of NASA/SDO and the AIA, EVE, and HMI science teams.

ing to Shallow sunspot model [5], which is the prevailing sunspot model. $5 \mathrm{Mm}$ is the largest stable sunspot with $\mathrm{B}_{o} \approx 2.6-2.7 \mathrm{kG}$ according to the model. In this article we will show multiple results regarding the sunspot, and the radio flares and and other types of activity it produced, at various radio frequencies measured with MRO radio telescopes. We will compare the results to previous studies presented in [6] and [7] and show that NOAA 12192's activity was exceptional as it produced several flares during the ten days of its visibility.

\section{Instrumentation}

Solar maps at $37 \mathrm{GHz}$ are produced with the 13.7-meter radio telescope, RT-14, which is a cassegain type antenna. RT-14 is located at Metsähovi Radio Observatory (MRO), Aalto University (Helsinki Region, Finland, GPS: N 60 13.04 E 24 23.35). The radio telescope provides full disk solar mapping, partial solar mapping, and, additionally, the ability to track any selected point on the solar disk. The beam size of the telescope is 2.4 arcmin at $37 \mathrm{GHz}$. The receiver is a Dicke type radiometer, thus, the radiometer's own noise will be filtered out. For the temperature stabilization of the receiver, a Peltier element is used. The noise temperature of the $37 \mathrm{GHz}$ receiver is around $280 \mathrm{~K}$, and the temporal resolution during the observations is $0.1 \mathrm{~s}$ or less. The radio emission comes from the solar chromosphere (transition region), and is recorded as intensity. More information about the Metsähovi RT-14 can be found in [7] and [8].

Metsähovi RT-1.8 is a radio telescope with a $1.8 \mathrm{~m}$ dish diameter dedicated for continuous solar observations. The telescope has a beam size of 81.6 arc min and its system noise temperature is $270 \mathrm{~K}$. It observes the total radiation of the Sun at a frequency of $11.2 \mathrm{GHz}$. The emission measured at $11.2 \mathrm{GHz}$ originates from lower corona. The Quiet Sun Level (QSL) is around 12,000 K at $11.2 \mathrm{GHz}$. The radiotelescope is used for observing solar radio bursts, as it acts as a detector of general solar activity. Also studies on solar oscillations have been done. High sampling rate $(5 \mathrm{kHz})$ enables studying flare fine structure, including short periodic oscillation phenomena. The radio telescope has no protective radome, therefore it is vulnerable to prevailing weather conditions. Around 200 solar radio bursts have been detected since its launch in 2001. Full documentation of the Metsähovi RT-1.8 can be found in [9]. The telescope has a logarithmic output which can be utilized in the case of strong bursts.

MRO joined the worldwide solar radio burst observing network, e-Callisto, in the fall of 2010 [10]. The network is coordinated by ETH Zürich and it consists of 69 instruments in 38 locations all over the world, covering solar radio spectral observations 24 hours a day throughout the year [11]. The network focuses on the decimeter and meter wavelength range. The first radio emission coming from the solar corona observed with the Callisto system was measured on September 24, 2010 [10]. The current frequency coverage in Metsähovi is 50 - 1450 $\mathrm{MHz}$ (the lower band is $50-846 \mathrm{MHz}$ and the higher band $790-1447.250 \mathrm{MHz}$ ). Due to the low instrumental sensitivity studies of the quiet Sun are not possible.

The changing radio emission indicates variation of solar magnetic activity. The birth of radio emission is af- 
fected by all plasma parameters, for example, temperature and density. This signifies the importance of radio observations and introduces interesting information on the subject. Gyromotion of thermal electrons in a presence of a magnetic field causes thermal gyroresonance emission. In active regions the magnetic field may have the strength to render the corona optically thick to enable gyroresonance absorption at the frequency range of 1 $18 \mathrm{GHz}$. Plasma emission is the most prevailing emission mechanism in solar flares up to $3 \mathrm{GHz}$ [12]. At mmwavelengths gyro-resonance emission above the sunspot [13] and free-free radiation from plasma in active region loops are found [14].

\section{Observations}

\subsection{Solar Radio Maps at $37 \mathrm{GHz}$}

Both variations in brightness and in size of the radio brightening can be derived from the MRO $37 \mathrm{GHz}$ observations. It should be noted, that the radio brightening located at the same location as the sunspot NOAA 12192. The $37 \mathrm{GHz}$ results are described in the two following subsections.

\subsubsection{Variation in Brightness}

Variation in brightness temperature of the radio brightening is listed in the Table 1 . The largest values are measured on October 26, 2014, when the brightness temperature is close to $200 \%$ compared to the Quiet Sun Level (QSL), which is $7800 \mathrm{~K}$. On other days within the duration of the radio brightenings activity the level is between about 120 and $140 \%$ compared to QSL.

\subsubsection{Variation in Size}

Four selected solar radio maps during October 20 - 29, 2014 when the NOAA 12192 sunspot was both active and visible, measured at $37 \mathrm{GHz}$ are shown in Figure 2. It can clearly be seen that the size of the radio brightenings varies with about the factor of 2 .

Table 1. Brightness temperature, in per cents compared to QSL, of the radio brightening measured at $37 \mathrm{GHz}$ from solar maps. Duration of each solar map scan is $7 \mathrm{~min}$.

\begin{tabular}{cccccc}
\hline Date & Start time & Temperature & Date & Start time & Temperature \\
\hline 20.10 .2014 & $09: 50: 24$ & 103.23 & 25.10 .2014 & $08: 59: 51$ & 137.69 \\
20.10 .2014 & $10: 00: 49$ & 124.06 & 25.10 .2014 & $09: 27: 19$ & 134.81 \\
20.10 .2014 & $10: 10: 48$ & 122.55 & 25.10 .2014 & $12: 16: 33$ & 125.48 \\
21.10 .2014 & $08: 40: 29$ & 119.81 & 26.10 .2014 & $10: 28: 44$ & 149.03 \\
22.10 .2014 & $08: 03: 19$ & 125.37 & 26.10 .2014 & $10: 59: 57$ & 199.07 \\
22.10 .2014 & $08: 21: 49$ & 125.83 & 26.10 .2014 & $11: 12: 50$ & 188.81 \\
22.10 .2014 & $11: 10: 11$ & 122.64 & 26.10 .2014 & $11: 22: 48$ & 176.08 \\
22.10 .2014 & $12: 04: 49$ & 121.93 & 26.10 .2014 & $11: 32: 13$ & 174.24 \\
23.10 .2014 & $10: 07: 36$ & 120.79 & 26.10 .2014 & $11: 49: 31$ & 188.87 \\
23.10 .2014 & $10: 18: 53$ & 119.78 & 28.10 .2014 & $10: 20: 27$ & 126.04 \\
23.10 .2014 & $12: 22: 47$ & 118.03 & 28.10 .2014 & $10: 28: 49$ & 128.41 \\
24.10 .2014 & $07: 31: 01$ & 121.77 & 28.10 .2014 & $11: 55: 21$ & 128.06 \\
24.10 .2014 & $07: 40: 02$ & 144.28 & 29.10 .2014 & $09: 16: 01$ & 128.06 \\
24.10 .2014 & $07: 50: 10$ & 145.07 & 29.10 .2014 & $09: 24: 23$ & 127.44 \\
24.10 .2014 & $07: 58: 58$ & 124.88 & 29.10 .2014 & $10: 48: 32$ & 118.22 \\
24.10 .2014 & $10: 43: 27$ & 124.43 & 29.10 .2014 & $10: 56: 53$ & 120.40 \\
24.10 .2014 & $11: 42: 48$ & 125.62 & & & \\
\hline
\end{tabular}



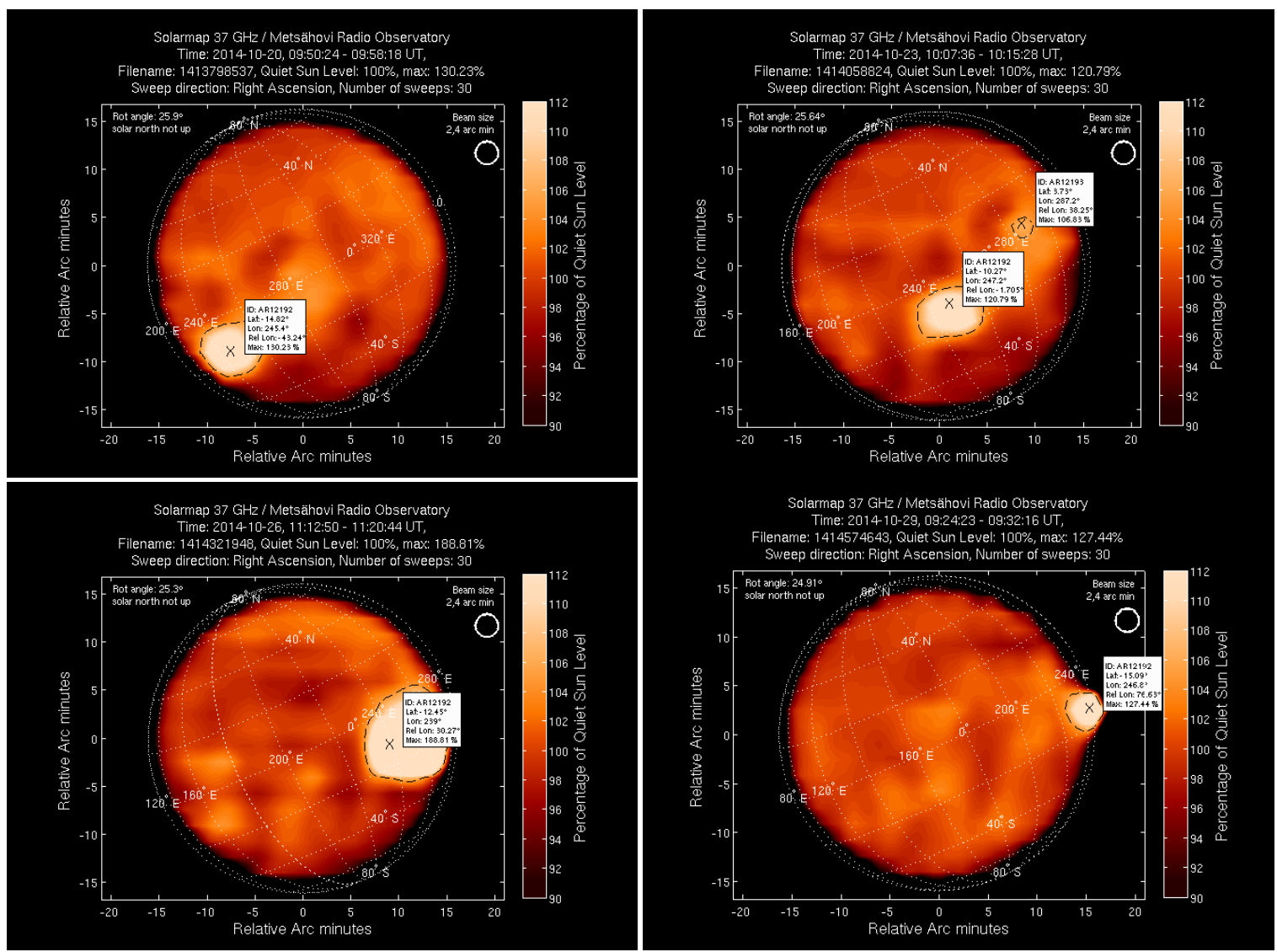

Figure 2. Solar radio maps measured on October 20, 23, 26 and 29, 2014.

\subsection{Measurements at $11.2 \mathrm{GHz}$}

We have listed all flares that exceed 100 Solar Flux Units (SFU) in Table 2. Peak flux values are estimations with errors approximately $\pm 10 \%$. An example of a solar radio burst measured with RT-1.8 on October 24, 2014 is in Figure 3. Observed solar radio bursts are associated with certain solar flares listed in Table 2. According to [15] about $20 \%$ of all X-class flares have strength of X2.0 - X2.9. When we take into account all possible flares, only less than $1 \%$ are X-class flares. The strongest of the now observed radio bursts at 2100 SFU is also rare in the long time series measured at MRO [6]. However, the previous time series did not include calibrated flux values.

\subsection{Measurements with Callisto}

In total, eight type III solar radio bursts listed in Table 3 were recorded at $200-400 \mathrm{MHz}$ band of the e-Callisto. Also some events were recorded at lower frequencies, which are not included in this study. An example of a Callisto recording measured on October 24, 2014 is shown in Figure 4. The radio bursts were also observed with several other e-Callisto stations all around the world [11].

\subsection{Comparison with Long Time Series Measured at MRO}

In comparison in [6], for example, in August, 2008, which was the most active month within the time series, 22 solar radio flares were measured. During other months in the time series, from January, 2000 to January, 2014, the number of flares exceeded ten only six times at the integration time of a month. During the ten days in October, 2014, 12 flares were measured at $11.2 \mathrm{GHz}$.

We also compared to the long time series study in [7], which includes data from 1978 to 2011 (solar cycles 21-14). In the study the strongest solar radio brightenings are $145 \%$ to QSL (11300 K). During October 26 , 
Table 2. Estimated maximum peak flux values at $11.2 \mathrm{GHz}$. Total of 12 flares were recorded at $11.2 \mathrm{GHz}$.

\begin{tabular}{|c|c|c|c|}
\hline Date & Time & Estimated peak flux (SFU) & Solar flare classification [4] \\
\hline 20.10 .2014 & $06: 36-06: 48$ & 150 & C6.3 \\
\hline 20.10 .2014 & 09:03 - 09:14 & 1600 & M3.9 \\
\hline 21.10 .2014 & $10: 28$ - 10:37 & 180 & \\
\hline 21.10 .2014 & $10: 48-10: 50$ & 170 & \\
\hline 21.10 .2014 & $12: 27-12: 29$ & 340 & $\mathrm{C} 4.4$ \\
\hline 21.10 .2014 & $13: 17-13: 20$ & 110 & \\
\hline 21.10 .2014 & $13: 37-13: 38$ & 940 & M1.2 \\
\hline 23.10 .2014 & 09:44 - 09:52 & 510 & M1.1 \\
\hline 24.10 .2014 & $07: 40-07: 46$ & 1600 & M4.0 \\
\hline 24.10 .2014 & 08:02 - 08:03 & 170 & \\
\hline 26.10 .2014 & $10: 34-12: 30$ & 2100 & $\mathrm{X} 2.0$ \\
\hline 27.10 .2014 & 09:20 - 11:00 & no calibration available & M6.7 \\
\hline
\end{tabular}

Table 3. Solar radio bursts, including the burst type, measured with the Callisto system (200 - $400 \mathrm{MHz})$.

\begin{tabular}{ccc}
\hline Date & Time & Type \\
\hline 21.10 .2014 & $10: 01: 11-10: 02: 13$ & III \\
21.10 .2014 & $10: 48: 01-10: 49: 45$ & III \\
21.10 .2014 & $13: 36: 02-13: 37: 57$ & III \\
23.10 .2014 & $11: 43: 48-11: 44: 00$ & III \\
24.10 .2014 & $07: 39: 34-07: 41: 43$ & III \\
24.10 .2014 & $08: 02: 04-08: 04: 35$ & III \\
24.10 .2014 & $10: 01: 36-10: 02: 05$ & III \\
26.10 .2014 & $10: 57: 37-10: 58: 05$ & III \\
\hline
\end{tabular}

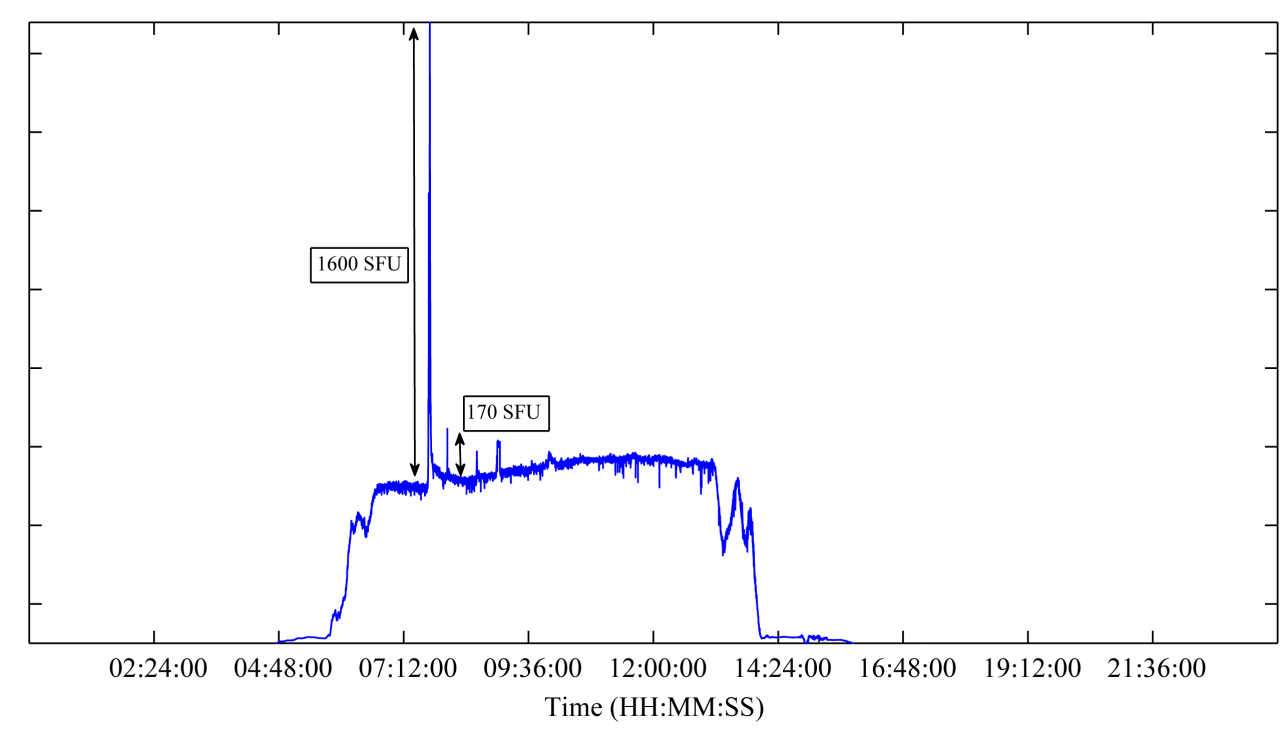

Figure 3. Two solar radio bursts on October 24, 2014 were measured at $11.2 \mathrm{GHz}$. The last wide spike is due to the calibration of the system. 


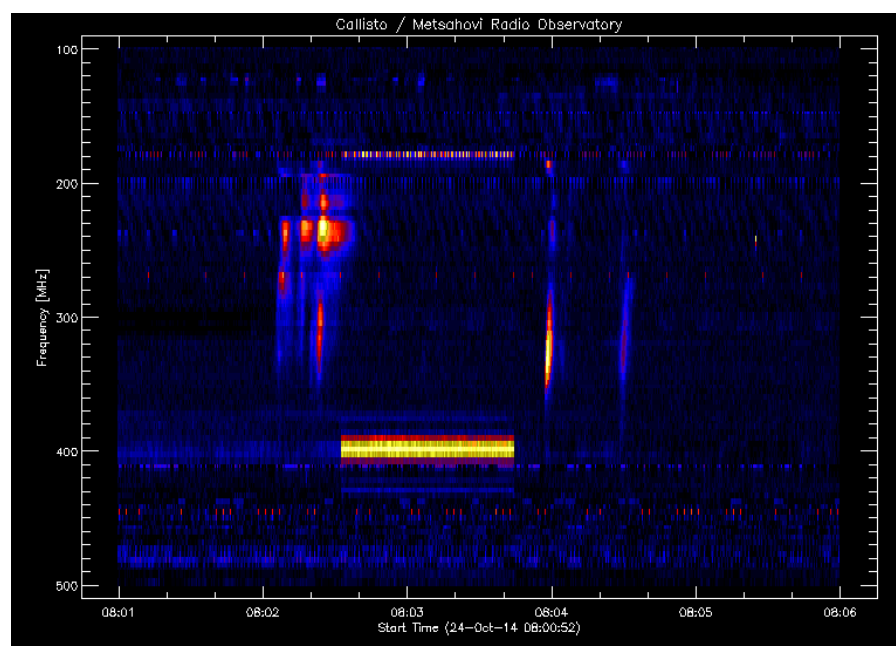

Figure 4. Type III solar radio burst measured with the Callisto system at $200-500 \mathrm{MHz}$ on October 24, 2014. The large horizontal anomaly at about $400 \mathrm{MHz}$ is due to RFI.

2014 145\% QSL limit was exceeded on six occasions, and on its strongest point it reached about 200\% QSL. The now recorded radio brightening belongs to the strongest category including less than $5 \%$ of radio brightenings ever measured at MRO.

\section{Conclusions}

In this article we have shown a review of radio signatures of NOAA 12192 measured at MRO at various instruments and radio frequencies. The radio brightening is remarkable even when the long time series measured at MRO are considered. The number of flares measured with RT-1.8 was 12 during the active period of the brightening, October 20 - 29, 2014, which makes this brightening one of the most active during the long time series ever measured at MRO. In comparison in [6], for example, in August, 2008, which was the most active month within the time series, 22 solar radio flares were measured. When the results were compared to the long time series [7], it can be concluded that the now recorded radio brightening belongs to the strongest category including less than $5 \%$ of radio brightenings ever measured at MRO.

This exceptional sunspot will lead to follow-up studies. For example, several of the events measured at 11.2 $\mathrm{GHz}$ revealed interesting double-peak structures. Further studies of the double-peak structures will be conducted by the authors in the near future.

\section{Acknowledgements}

NOAA 12192 image created using the ESA and NASA funded Helioviewer Project.

\section{References}

[1] Kiess, C., Rezaei, R. and Schmidt, W. (2014) Properties of sunspot umbrae observed in cycle 24. Astronomy and Astrophysics, 565, Article ID: AA52. http://dx.doi.org/10.1051/0004-6361/201321119

[2] Schad, T.A. and Penn, M.J. (2010) Structural Invariance of Sunspot Umbrae over the Solar Cycle: 1993-2004. Solar Physics, 262, 19-33. http://dx.doi.org/10.1007/s11207-009-9493-8

[3] Zharkov, S., Zharkova, V.V. and Ipson, S.S. (2005) Statistical Properties of Sunspots in 1996-2004: I. Detection, NorthSouth Asymmetry and Area Distribution. Solar Physics, 228, 377-397. http://dx.doi.org/10.1007/s11207-005-5005-7

[4] NOAA’s Space Weather Prediction Center's Web Site. http://www.swpc.noaa.gov/

[5] Solov'ev, A. and Kirichek, E. (2014) Basic Properties of Sunspots: Equilibrium, Stability and Long-Term Eigen Oscillations. Astrophysics and Space Science, 352, 23-42. http://dx.doi.org/10.1007/s10509-014-1881-3

[6] Kallunki, J. and Uunila, M. (2014) Total Solar Flux Intensity at $11.2 \mathrm{GHz}$ as an Indicator of Solar Activity and Cyclicity. 
International Journal of Astronomy and Astrophysics, 4, 437-444. http://dx.doi.org/10.4236/ijaa.2014.43039

[7] Kallunki, J., Lavonen, N., Järvelä, E. and Uunila, M. (2012) A Study of Long-Term Solar Activity at 37 Ghz. Baltic Astronomy, 21, 255-262.

[8] Kallunki, J. (2013) Studies of Solar Activity with Emphasis on Quasi-Periodic Oscillations. Doctoral Thesis, University of Turku, Ser. A I Tom. 467, Turku.

[9] Kallunki, J. (2009) Possibilities of the Metsähovi Radiotelescopes for Solar Observations. Licenciate Thesis, Faculty of Information and Natural Sciences, Helsinki University of Technology, Helsinki.

[10] Kallunki, J., Uunila, M. and Monstein, C. (2013) Callisto Radio Spectrometer for Observing The Sun-Metsähovi Radio Observatory Joins the Worldwide Observing Network. IEEE Aerospace and Electronic Systems Magazine, 28, 5-9. http://dx.doi.org/10.1109/MAES.2013.6575404

[11] e-Callisto Web Site (2014) International Network of Solar Radio Spectrometers. http://www.e-callisto.org/

[12] Benz, A. (2006) Radio Emission of Solar Flare Particle Acceleration. 6th International Workshop on Planetary and Solar Radio Emissions, Graz, 20-22 April 2005, 325-338.

[13] Shibasaki, K., Enome, S., Nakajima, H., et al. (1994) A Purely Polarized S-Component at 17-GHz. Publications of the Astronomical Society of Japan, 46, L17-L20.

[14] Shibasaki, K., Alissandrakis, C.E. and Pohjolainen, S. (2011) Radio Emission of the Quiet Sun and Active Regions (Invited Review). Solar Physics, 273, 309-337. http://dx.doi.org/10.1007/s11207-011-9788-4

[15] Le, G., Yang, X., Liu, Y., et al. (2014) Statistical Properties of X-Class Flares and Their Relationship with Super Active Regions during Solar Cycles 21-23. Publications of the Astronomical Society of Japan, 350, 443-447. 
Scientific Research Publishing (SCIRP) is one of the largest Open Access journal publishers. It is currently publishing more than 200 open access, online, peer-reviewed journals covering a wide range of academic disciplines. SCIRP serves the worldwide academic communities and contributes to the progress and application of science with its publication.

Other selected journals from SCIRP are listed as below. Submit your manuscript to us via either submit@scirp.org or Online Submission Portal.
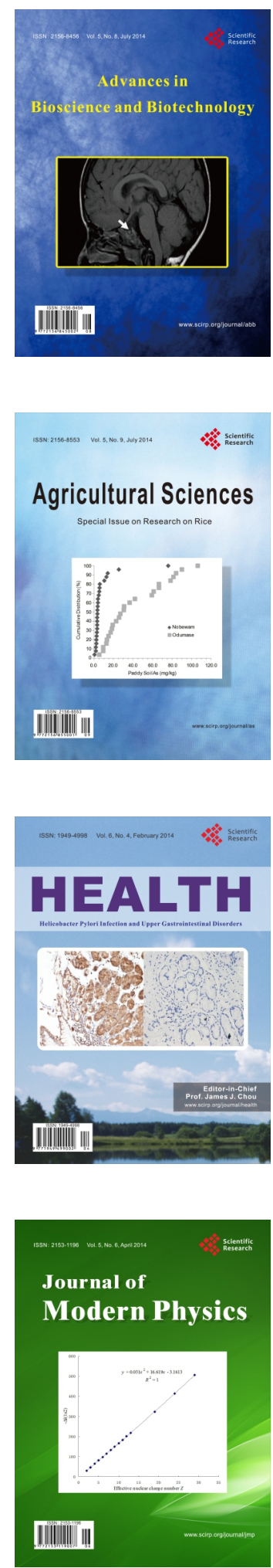
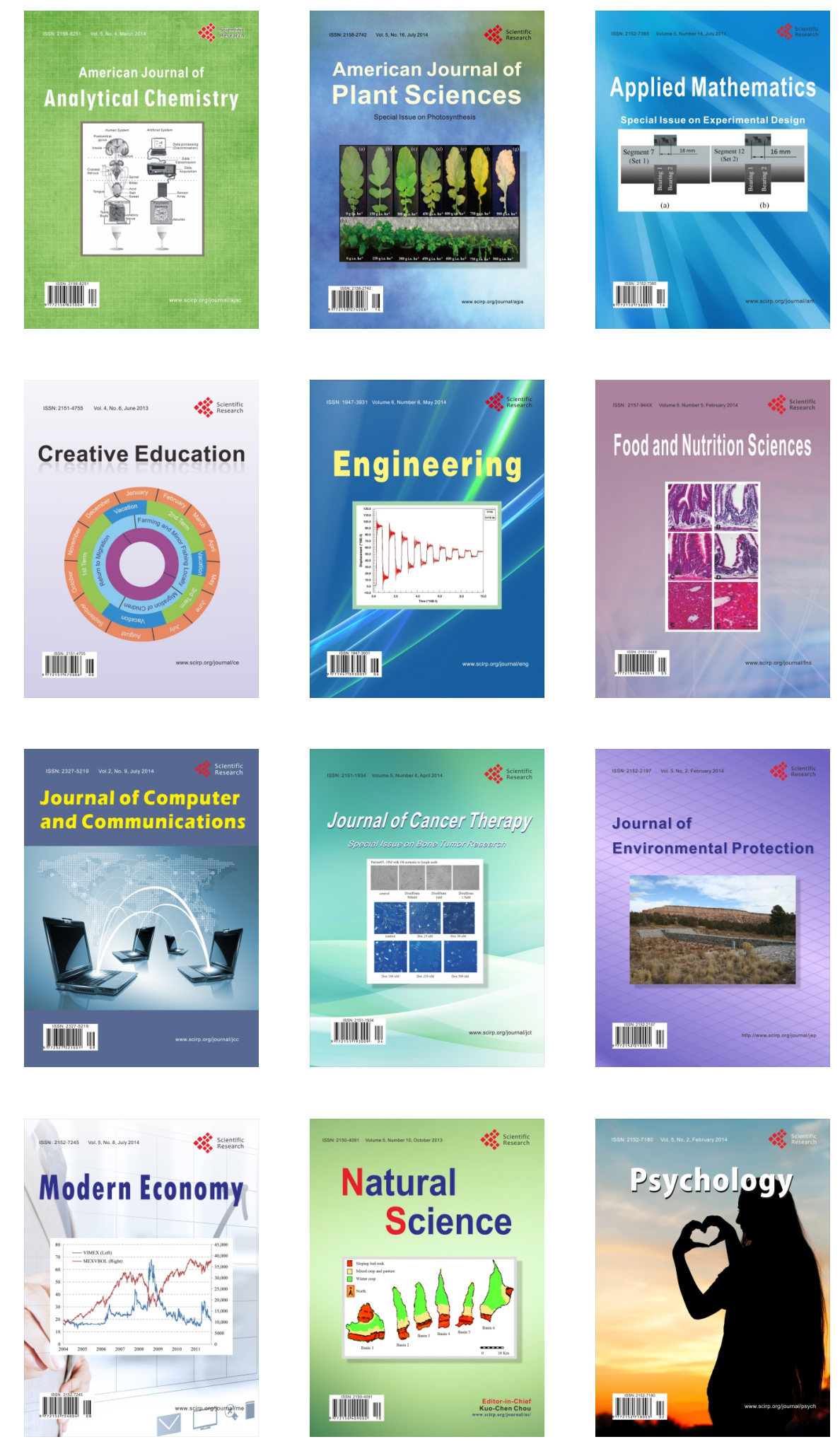